\title{
Erratum to: Towards an Optical Internet
}

\author{
Admela Jukan \\ Institute of Communication Networks, \\ Vienna University of Technology, Austria
}

\section{Erratum to:}

\section{A. Jukan (Ed.) \\ Towards an Optical Internet \\ DOI: $10.1007 / 978-0-387-35491-0$}

The book was inadvertently published with an incorrect name of the copyright holder. The name of the copyright holder for this book is: (c) IFIP International Federation for Information Processing. The book has been updated with the changes. 